\title{
Einwanderer als Wähler, Gewählte und transnationale Akteure
}

\author{
Karen Schönwälder
}

Zusammenfassung: Die politische Inkorporation von Einwanderern ist in Deutschland ein relativ neues, noch wenig bearbeitetes Forschungsfeld. Dieser Literaturbericht stellt ausgewählte neuere Ergebnisse der internationalen Forschung zur politischen Partizipation von Einwanderern und Angehörigen ethnischer Minderheiten vor. Neben einigen Kernthemen der Forschung zu den USA werden die Rolle institutioneller „Motoren der Inkorporation“, das Vordringen von Einwanderern in die Parlamente, Protestaktionen von Ausländerinnen und Ausländern sowie Erkenntnisse zu transnationalen Formen politischen Engagements diskutiert.

Schlagwörter: Einwanderer $\cdot$ politische Partizipation $\cdot$ politische Repräsentation $\cdot$ transnationales Engagement

Abstract: The political incorporation of immigrants is a relatively new and little researched topic in Germany. This review article presents selected results of recent international research on the political participation of immigrants and members of ethnic minorities. Apart from some core themes of research on the United States, I discuss the role of institutional motors of incorporation, the emergence of parliamentarians with a migration background, protest activities of non-citizens, and some findings on transnational forms of political engagement.

Keywords: immigrants $\cdot$ political participation $\cdot$ political representation $\cdot$ transnational engagement

(C) VS-Verlag 2009

Karen Schönwälder $(\bowtie)$

Max-Planck-Institut zur Erforschung multireligiöser und multiethnischer Gesellschaften

Hermann-Föge-Weg 11, 37073 Göttingen

E-Mail: schoenwaelder@mmg.mpg.de 


\section{Einleitung}

In den USA werden ab 2042 die sogenannten Hispanics gemeinsam mit den AfroAmerikanern und anderen nicht als ,weiß“ klassifizierten Amerikanern die Mehrheit der Bevölkerung stellen. ${ }^{1}$ Schon in den letzten Jahren ist das Gewicht der Stimmen gerade der sogenannten Hispanics in den Präsidentschaftswahlen deutlich gewachsen, zum Teil dadurch, dass Staaten mit großen Einwandereranteilen auch durch die zeitliche Abfolge der Vorwahlen eine entscheidende Bedeutung erhielten (Frey 2008: 2; vgl. auch Werz 2008). Kein Wunder, dass die politischen Einstellungen und Verhaltensweisen von Einwanderern - bzw. von Angehörigen ethnischer Minderheiten - in Nordamerika ein etablierter Forschungsgegenstand sind. Selbst in den USA allerdings beklagen in diesem Feld ausgewiesene Wissenschaftler, dass die Erforschung der politischen Inkorporation von Einwanderern hinter der der ökonomischen und sozialen Inkorporation zurückbleibe. Während politische Parteien und andere Institutionen in den 1980er Jahren angefangen hätten, mögliche politische Konsequenzen der steigenden Einwandererzahlen zu beobachten, greife die Politikwissenschaft erst langsam die neuen Fragen auf: „the study of political behaviour among immigrants is still in relative infancy“" (Ramakrishnan 2005: 14; ähnlich de la Garza 2004: 91).

Auch in Deutschland entwickelt sich das Interesse am politischen Verhalten der Eingewanderten und insbesondere der Eingebürgerten erst langsam und in Reaktion auf deren sich abzeichnende wahlpolitische Relevanz. Sollte der Trend bei den Einbürgerungszahlen anhalten, wird das wahlpolitische Gewicht der Deutschen mit Migrationsgeschichte nur langsam zunehmen. ${ }^{2}$ Dennoch könnten vor allem bei knappen Wahlentscheidungen sowie bei Wahlen in einigen Städten und Bundesländern die Eingebürgerten und andere Deutsche aus Migrantenfamilien bald eine wichtige Rolle spielen. ${ }^{3}$ Das Interesse an Erkenntnissen über ihre politischen Präferenzen und Loyalitäten wird dementsprechend wachsen. Diese äußern sich natürlich nicht nur bei Wahlen. Migrant(inn)en werden nicht erst mit Erwerb der Wahlrechte, also in der Regel mit der Einbürgerung, politisch aktiv, und ihre politische Partizipation beschränkt sich - ob Ausländer(innen) oder Deutsche - nicht auf die Beteiligung an Wahlen.

Dieser Literaturbericht betrachtet dementsprechend einige ausgewählte neuere Erkenntnisse der Forschung zu unterschiedlichen Formen und wichtigen Bedingungen politi-

1 Vgl. Presseberichte im August $2008 \mathrm{zu}$ den neuesten Hochrechnungen auf der Basis von Volkszählungsdaten. Der Ausdruck „Hispanics“ steht für eine sehr heterogene Gesamtheit der Bevölkerung aus den spanisch- (und portugiesisch-)sprachigen Ländern Lateinamerikas.

2 Laut dem Mikrozensus 2006 gibt es 7,84 Millionen Deutsche mit Migrationshintergrund, von denen über 5 Millionen im wahlberechtigten Alter sind. Diese Gruppe enthält eingebürgerte Deutsche, zumeist Einwanderer der ersten Generation, einige, aber keineswegs alle Aussiedler sowie Kinder auch bi-nationaler Elternpaare, die durch ein deutsches Elternteil bei Geburt deutsche Staatsangehörige sind. Der erste Jahrgang von Einwandererkindern ohne deutsche Eltern, die aufgrund ihrer Geburt in Deutschland Deutsche wurden, wird 2018 wahlberechtigt sein; ihre Zahl wird auf etwa 40000 (pro Geburtsjahrgang) geschätzt. Jährlich wurden zuletzt etwa 113-140 000 Ausländer(innen) eingebürgert.

3 Dies gilt etwa für Stuttgart, München oder Frankfurt a. M., also Großstädte mit Ausländerzahlen von über 20 Prozent. In Stuttgart und Frankfurt a. M. waren 2005 nach dem Mikrozensus jeweils etwa 15 Prozent der Bevölkerung Deutsche mit Migrationshintergrund. 
scher Partizipation. Dabei werden zunächst einige grundlegende Erkenntnisse der neueren US-Forschung vor allem zur Partizipation bei Wahlen vorgestellt. Anschließend werden mit der Rolle institutioneller „Motoren der Inkorporation“, dem Vordringen von Einwanderern in die Parlamente und mit Protestaktionen von Ausländer(inne)n zentrale Bedingungen bzw. Formen der Inkorporation von Migrant(inn)en in die politischen Strukturen und Prozesse der Einwanderungsländer diskutiert. Abschließend wird eine erste Bilanz der Forschung vorgenommen, die sich mit transnationalen Formen politischen Engagements befasst.

\section{Gar nicht so anders, aber doch verschieden: Erkenntnisse zu den USA}

Die USA sind wohl das Land, für das die politische Partizipation der Migranten am besten erforscht ist. Auch hier allerdings heben viele Forscher eklatante Kenntnislücken hervor. Diese betreffen vor allem die bislang keineswegs gängige Unterscheidung zwischen Einwanderern und Angehörigen einer ethnischen Gruppe, also die spezifische Untersuchung des Einflusses von migrationsbedingten Besonderheiten, sowie die breitere Erforschung der verschiedenen Herkunfts- und ethnischen Gruppen, von denen bislang insbesondere die African Americans und die Latinos bzw. Hispanics besser untersucht sind. Letzteres ist dann wichtig, wenn man annimmt, dass das Herkunftsland, eine gruppenspezifische Migrationsgeschichte oder ethnische Besonderheiten die politische Partizipation beeinflussen. Trotz dieser Lücken gibt es aber einen breiten Konsens über einige Haupttrends in der politischen Partizipation von Einwanderern und ihren Kindern:

Zunächst zeigen die vorliegenden empirischen Ergebnisse recht deutlich, dass Einwanderer sich in ihrem politischen Verhalten nicht fundamental von anderen USAmerikaner(inne)n unterscheiden: Gängige Prädiktoren politischen Engagements treffen auch auf sie zu. Eine höhere Bildung, ein höheres Einkommen und Alter sind auch bei Einwanderern mit einer höheren politischen Partizipation verknüpft (vgl. bilanzierend Ramakrishnan 2005: 45-50). Dies bedeutet gleichzeitig, dass das sozio-demographische Profil der Eingewanderten niedrigere politische Beteiligungsraten begünstigt wenn nicht besondere mobilisierende Faktoren dem entgegenwirken. Tatsächlich sind die Partizipationsraten eingebürgerter US-Amerikaner niedriger als die anderer USBürger(innen): ,in contemporary elections, most empirical studies indicate that naturalized citizens register and vote at rates lower than comparably situated U.S.-born citizens" (DeSipio 2001: 81; vgl. auch de la Garza 2004). Dabei besteht unter den Eingewanderten selbst in der dritten Generation noch eine Spanne zwischen 43 (Latinos) und 63 Prozent (Weiße) Wahrscheinlichkeit der Wahlbeteiligung (Ramakrishnan 2005: 85).

Über andere Partizipationsformen als die Wahlbeteiligung gibt es weniger Daten; Ramakrishnan (2005: 144-160) konstatiert, dass bei bestimmten Aktivitäten, wie dem Verfassen von Briefen an Politiker oder dem Einsatz in politischen Parteien, auch nach statistischer Kontrolle des Einflusses von Alter, Geschlecht und sozioökonomischem Status eine niedrigere Partizipation der Einwanderer und der Angehörigen von Minderheiten festzustellen ist, dass es aber auch Aktionsformen gibt - wie die Teilnahme an 
örtlichen Versammlungen -, an denen etwa Latinos besonders stark beteiligt sind. Ansonsten aber ist die Hierarchie der (abgefragten) Aktionsformen bei allen Gruppen (hier: Hispanics, Asians, Whites, Blacks) sehr ähnlich, Unterschiede sind auffälliger im Niveau als in der Art der Beteiligung. Dennoch bleibt festzuhalten, dass - wenn die USA beispielhaft sind - auch bei einer relativ offenen Einbürgerungspolitik von über Jahrzehnte anhaltenden Unterschieden in der politischen Partizipation von Bürger(inne)n mit bzw. ohne Migrationshintergrund auszugehen ist.

Um die bestehenden Unterschiede umfassend zu erklären - und dies ist die zweite Grunderkenntnis -, müssen migrationsbezogene und gruppenspezifische Faktoren herangezogen werden, die offenbar mittelfristig wirksam bleiben. Hierunter sind offensichtlichere Faktoren wie die Aufenthaltsdauer im Land ${ }^{4}$ und das Niveau der Englischkenntnisse (z.B. für Mexikaner Barreto/Muñoz 2003: 441). Weniger eindeutig ist, in welchem Maß und wie die politische Sozialisation im Herkunftsland und dessen kulturelle Traditionen (z.T. über Generationen hinweg) Einfluss nehmen. ${ }^{5}$ Zum Teil werden auch Einflüsse einer gruppenspezifischen politischen Sozialisation in den USA selbst vermutet. So formuliert de la Garza die Hypothese, Latinos würden weniger und anders wahlpolitisch sozialisiert, hätten auch zumeist weniger Kontakt mit dem Staat und zentralen gewählten Institutionen als in den USA geborene patriotische US-Amerikaner (de la Garza 2004: 101). Eine umfassende empirische Überprüfung solcher Annahmen und damit eine befriedigende Erklärung beobachteter Unterschiede vor allem im wahlpolitischen Verhalten liegt bislang nicht vor (so de la Garza 2004: 101; ähnlich für Asian Americans Wong et al. 2008: 88).

Eine Präferenz für individualistische Ansätze und quantitative Analysen sowie ein häufig auf den nationalen Kontext der USA konzentrierter Blick tragen dazu bei, dass der Einfluss nationalstaatlicher institutioneller Kontexte sowie spezifischer Institutionen und kollektiver Akteure auf Niveau und Formen der politischen Partizipation von Einwanderern und ihren Nachkommen in den USA weniger umfassend untersucht wird (vgl. aber z. B. Skerry 2005; Jones-Correa 2007). Solchen Bedingungen kommt aber nun einer neueren vergleichenden Studie zu den USA und Kanada zufolge - eine zentrale Rolle für die politische Mobilisierung von Einwanderern in den Strukturen des Aufnahmelandes zu. Folgt man Irene Bloemraad (2006: 102-106), dann sind es gezielte Interventionen seitens des Staates - und nicht lediglich „Gelegenheitsstrukturen“ -, die Muster der politischen Inkorporation verändern können. Ihr Ausgangspunkt ist die Beobachtung, dass im Hinblick auf Einbürgerungsquoten und auf die Anteile von Einwanderern (hier definiert als im Ausland geborene Personen) in den Parlamenten zwischen den USA und Kanada deutliche Unterschiede bestehen. Tatsächlich ist heute in den USA nur gut ein Drittel der im Ausland geborenen Bevölkerung zu Staatsbürgern der USA geworden, in Kanada dagegen galt Entsprechendes für 70 Prozent der foreign born (Bloemraad 2006: 17-18). Auch bezüglich des Anteils der Einwanderer in den

4 Ramakrishnan findet für die erste Einwanderergeneration durchweg mit längerem Aufenthalt eine höhere Wahlbeteiligung. Im Vergleich der 1., 2. und 3. Generation gibt es aber Unterschiede zwischen den ethnischen Gruppen (2005: 80-81).

5 Hierzu gehören auch Folgen unterschiedlichster politisch-kultureller Beziehungen zum Einwanderungsland. So wird die hohe politische Partizipation der Filipinos mit der intensiven Intervention der USA in den Philippinen erklärt, vgl. Ramakrishnan 2005: 97. 
nationalen Parlamenten konstatiert Bloemraad beträchtliche Unterschiede (2006: 5664). Solche Diskrepanzen seien erst seit den 1960er bzw. 1970er Jahren entstanden und hätten - so ihre These - ihre Grundlage in den unterschiedlichen Integrations- und Diversitätspolitiken beider Länder.

Methodisch ist es schwierig, die Wirkungen komplexer politischer Interventionen nachzuweisen. Aussagen über die Effekte einer multikulturellen oder auch einer assimilationsorientierten Politik beruhen denn auch oft auf Beobachtungen allgemeiner Korrelationen von z.B. einer deklarierten Regierungspolitik und Arbeitslosenraten oder Einbürgerungsquoten, ohne dass geklärt wird (oder geklärt werden kann), ob tatsächlich die jeweilige Migrations- und Integrationspolitik und nicht etwa ganz andere Faktoren ursächlich sind. Zum Teil werden denn auch konträre Thesen zu den Wirkungen solcher Politiken vertreten. So schreiben Koopmans et al. (2005) der multikulturellen Politik (hier: der Niederlande) vor allem negative Wirkungen zu und glauben, dass die Betonung kultureller Differenz gerade nicht die politische Inkorporation, sondern einen Rückzug der Migrantengemeinschaften förderte, die ihre Aktivitäten vor allem nach innen ausrichteten (2005: 80). ${ }^{6}$ Auch Bloemraad kann letztlich nicht schlüssig nachweisen, dass die kanadische Politik des Multikulturalismus tatsächlich für relative hohe Einbürgerungsraten und eine relativ große Zahl von Einwanderern in den Parlamenten verantwortlich ist, also im Sinne einer Förderung der Partizipation in den etablierten Institutionen des Einwanderungslandes wirkt. Sie überprüft allerdings, ob Unterschiede zwischen Kanada und den USA eher mit den Aufenthaltszeiten und Hintergründen der Migranten erklärt werden könnten, und findet hierfür keine schlüssigen Anzeichen. Und sie entwickelt Thesen dazu, wie im Einzelnen staatliche Politik politische Partizipation fördern könnte, wobei sie drei Faktoren unterscheidet:

„Government policies can facilitate incorporation by providing immigrant communities with material resources to engage in political mobilization, by increasing access to political decision makers, and by shaping understandings of immigrants' symbolic place within the polity." (Bloemraad 2006: 106)

Der kanadische Multikulturalismus, wie er seit den 1970er Jahren entwickelt wurde, stelle genau diese materiellen und symbolischen Ressourcen bereit, derer sich Migrant(inn)en dann bedienen könnten; er legitimiere die ethnische Vielfalt und Ansprüche von Migrant(inn)en. Nicht zuletzt böten die institutionellen Strukturen des Multikulturalismus Kontexte, in denen Einwanderer Kontakte zum Staat und zu politischen Akteuren erhielten und politisch in den Strukturen und Prozessen des Aufnahmelandes sozialisiert würden. Der passive Ansatz der US-Politik, die aktive Integrationspolitik im Grunde nur für Flüchtlinge betreibe, biete solche materiellen und symbolischen Ressourcen sowie spezifischen Sozialisationskontexte nicht.

6 Empirische Basis ist die Auswertung der Berichterstattung einer nationalen Tageszeitung. In diesem Fall geht es um die Erklärung des Befundes, dass in den Niederlanden relativ wenig über Akteure berichtet wurde, die Migrant(inn)en und ethnische Minderheiten repräsentieren. Nicht berücksichtigt wurde dabei die empirische Beobachtung, dass in den Niederlanden relativ viele Abgeordnete einen Migrationshintergrund haben. 


\section{Vermittelnde Instanzen der politischen Inkorporation}

Wäre demnach der Staat - gegebenenfalls gemeinsam mit einem semistaatlichen Netzwerk aus Institutionen, die sich mit der Umsetzung von Integrations- oder multikultureller Politik befassen - heutzutage ein entscheidender "Motor der Inkorporation"? Die Frage, welche Rolle heute bestimmte intermediäre Institutionen für die politische Inkorporation von Einwanderern spielen, welche Rolle überhaupt institutionellen Strukturen als Grundlage oder auch Hindernis gleichberechtigter Partizipation zukommt, gehört zu den großen, in vielerlei Hinsicht offenen Forschungsthemen. In Europa hat sich die Aufmerksamkeit bislang stark auf die Einbürgerungsbedingungen konzentriert. In der Tat liegt es auf der Hand, dass die Einbürgerung ehemaliger Ausländer(innen) eine breitere Teilnahme ermöglicht, und eine großzügige Einbürgerungspolitik darüber hinaus Offenheit für die Beteiligung der Einwanderer signalisieren und diese dadurch auch symbolisch stimulieren kann. Einen linearen Zusammenhang zwischen Einbürgerung und Partizipation aber gibt es offenbar nicht, wie Forschungsergebnisse zu den USA und auch Unterschiede, die sich zwischen europäischen Staaten und zwischen Migrantengruppen beobachten lassen, nahelegen. Insgesamt konstatieren Bauböck u. a. (2006: 63), dass über die konkreten Konsequenzen der Einbürgerung u. a. für politische Einstellungen und politisches Verhalten kaum Erkenntnisse vorliegen. In der Bundesrepublik wurde eine große Chance vertan, als man darauf verzichtete, mit Einführung des neuen Staatsangehörigkeitsrechts im Jahr 2000 dessen Wirkungen durch eine groß angelegte empirische Studie zu verfolgen.

Eine zentrale Rolle in der politischen Inkorporation der Einwanderer wurde etwa in den USA lange den Migrantenorganisationen zugeschrieben - dies in deutlichem Kontrast zur in Deutschland dominierenden Wahrnehmung dieser Organisationen als Hindernis einer Integration im Einwanderungsland. So gehen etwa Portes et al. (2008: 2-3) davon aus, dass Einwanderer nur im Ausnahmefall einen individuellen Zugang zur amerikanischen Politik finden; sie würden vielmehr durch Aktivisten mobilisiert. Dies seien bislang Aktivisten der „,ethnischen“ Organisationen gewesen: „Ethnic political organization has been the requisite first step of incorporation, as immigrants and their offspring learned to become Americans by first being ,ethnics““. Nun wird aber zunehmend gefragt, ob dies weiterhin (wenn überhaupt) zutreffend ist oder aber ob sich die Bedeutung von Migranten- bzw. ethnischen Organisationen grundlegend verändert. Zivilgesellschaftliche Mobilisierung erfolge immer mehr in Form von durch professionelle Eliten organisierte Kampagnen. Das Interesse der NGOs an der Entwicklung einer breiten Mitgliedschaft könne im Zuge dessen zurückgehen (DeSipio 2001, unter Verweis auf Skerry; vgl. ähnlich, bezogen auf Österreich, Waldrauch/Sohler 2004: 281). Die Selbstorganisationen könnten infolge dessen ihre (angenommene) Rolle als Sozialisationsagenturen für eine breite Gruppe von Migranten verlieren, gleichzeitig aber eine neue Rolle als Sozialisationsagenturen einer kleinen Führungsschicht erhalten, die über Funktionen in „ethnischen“ oder Migrantenorganisationen Zugang zu politischen Erfahrungen und Netzwerken gewinnen und auf dieser Basis auch in anderen Institutionen Karriere machen könnte.

In Europa hat vor allem Jean Tillie (2004; Fennema/Tillie 2001) Forschungen durchgeführt bzw. angestoßen, die die (an Putnam angelehnte) These überprüfen, dass ethnische 
Organisationen soziales Kapital repräsentieren, das sich in Form einer erhöhten Partizipation in den Institutionen und Prozessen des Aufnahmelandes auswirkt. Die Hypothesen lauten im Einzelnen, dass im institutionellen Netzwerk einer ethnischen Community Ressourcen enthalten seien, die das soziale Kapital der Individuen erhöhen. Über die Mitgliedschaft in Organisationen werde zudem soziales Vertrauen hergestellt, das wiederum in politisches Vertrauen umgewandelt werden könne und sich dann auf die wahlpolitische Partizipation förderlich auswirke (Tillie 2004: 534). Eine hohe Zahl ethnischer Organisationen und deren Vernetzung untereinander wurden hier als Ausdruck umfangreichen sozialen Kapitals (Zugang zu Ressourcen und Kontakten auch indirekt vermittels Vernetzung) interpretiert. Empirisch konnte für Amsterdam gezeigt werden, dass Unterschiede zwischen Migrantengruppen in der politischen Partizipation mit einem unterschiedlichen Organisationsgrad in Selbstorganisationen korrelieren (Tillie 2004). Für Brüssel (Jacobs et al. 2006a) und im Vergleich von Amsterdam und Berlin allerdings konnten eindeutige Zusammenhänge zwischen Zahl und Vernetzung ethnischer Organisationen und dem Partizipationsniveau der Individuen der betreffenden Herkunftsgruppe nicht nachgewiesen werden. Offenbar wird soziales Kapital nicht wie angenommen akkumuliert, es wirkt nicht in dieser Weise auf die politische Partizipation ein, oder solche Wirkungen hängen von weiteren Kontextfaktoren und Charakteristika der Organisationen und Netzwerke ab. Darüber hinaus ist das relative Gewicht von individuellen Charakteristika und Gruppenmerkmalen (wie eben der ethnischen Institutionen) weiter umstritten (Jacobs et al. 2006a; Jacobs/Tillie 2008).

Insgesamt ist die Literatur zur spezifischen Bedeutung einzelner kollektiver Akteure und institutioneller Kontexte für die politische Inkorporation und Mobilisierung von Migrant(inn)en sehr spärlich. Über die Gewerkschaften etwa, die in verschiedenen europäischen Ländern insofern eine herausragende Rolle einnehmen, als sie in bedeutendem Umfang Migrant(inn)en organisieren, gibt es kaum umfassende, befriedigende Studien (vgl. Penninx/Roosblad 2000; Öztürk 2002; Kühne et al. 1994). Gerade die weiteren Zusammenhänge zwischen gewerkschaftlicher Organisierung und in breiterem Sinne politischer Integration im Einwanderungsland oder die Rolle der Gewerkschaften für die Herausbildung politischer Führungskräfte sind weitgehend unerforscht. Über politische Parteien liegen kaum detaillierte Erkenntnisse vor. Auch religiöse Gemeinschaften könnten eine wichtige Rolle für die politische Sozialisation und Inkorporation spielen, sofern sie Räume politischer Kommunikation und Meinungsbildung sowie Orte, an denen eine Rekrutierung in politische Netzwerke stattfindet, sind und Gelegenheit bieten, relevante Fertigkeiten zu erlernen (so etwa Wong et al. 2008: 73, unter Verweis auf Jones-Correa und Leal). Hier bietet sich für die Zukunft ein weites offenes Forschungsfeld.

7 Berger und Koopmans (2004) ermittelten für Türken in Berlin höhere Werte für einige Aspekte politischer Partizipation und politischen Vertrauens als in Amsterdam, obwohl die Organisationsdichte und Vernetzung zwischen den Organisationen in Amsterdam größer waren. 


\section{Bedingungen parlamentarischer Karrieren}

Typischer als Studien zu einzelnen Institutionen sind Untersuchungen der allgemeinen Bedingungen, die die Entwicklung einer Bewegung oder auch den Aufstieg von parlamentarischen Repräsentanten der Einwanderung begünstigen (bzw. behindern). Sehr populär ist dabei in Europa die Berufung auf einen „opportunity structures approach“, wobei dies allerdings gelegentlich weniger ein anspruchsvolles theoretisches Konzept als vor allem die Bekräftigung einer verbreiteten Erkenntnis ist. Dass die Geschichte von Menschen gemacht wird, die aber im Rahmen vorgegebener Umstände handeln, wusste schließlich schon Karl Marx. Fruchtbar erscheinen vor allem Überlegungen, die sich auf das Zusammenwirken allgemeiner politisch-institutioneller Strukturen, politisch-kultureller Bedingungen (wie längerfristig etablierte Haltungen zur Stellung von Einwanderern bzw. ethnischen Minderheiten in der Gesellschaft) und der in konkreten Konstellationen vorliegenden Bedingungen, Motive und Interessen konzentrieren. Dies sollte (verstärkt) verknüpft werden mit einer Berücksichtigung der Charakteristika der Einwanderer selbst. So zeigt etwa Romain Garbaye (2005) in einer Studie zur Vertretung von Einwanderergruppen in kommunalen Parlamenten britischer und französischer Städte, wie in Großbritannien mehrere Faktoren zusammenwirkten, um in bestimmten Städten eine vergleichbar frühe Öffnung für Repräsentanten ethnischer Minderheiten zu bewirken. Dies waren vor allem das relative Mehrheitswahlsystem in Einpersonenwahlkreisen (das Wahlerfolge konzentriert siedelnder Gruppen erleichtert), die große Bedeutung des Antirassismus in der politischen Kultur und die zeitweilige Linksentwicklung der Labour Party, die eine multikulturelle Politik auch als Abgrenzungsmerkmal zu den von Thatcher und dann von Major geführten Konservativen einsetzte. In Birmingham etwa wurde 1993 eine zum Bevölkerungsanteil proportionale Vertretung der „ethnic minorities“ erreicht. Schon 1979 waren dort die ersten beiden ethnischen Minderheiten zugerechneten Councillors gewählt worden. In Frankreich dagegen konnten politische Strukturen, die den Bürgermeistern und lokalen politischen Eliten relativ großen Einfluss auf die Kandidatenauswahl geben, für eine Vertretung von Einwanderern sowohl blockierend als auch begünstigend wirken. Letzteres war der Fall, als sich dominierende Interpretationsmuster der französischen Gesellschaft veränderten und die Aufweichung lange strikt assimilationistischer Positionen eine gezielte Minderheitenpolitik akzeptabel machte. Nach ersten Durchbrüchen in den vorangegangenen Jahren wurde Garbaye (2005) zufolge ab ca. 2001 eine stärkere Öffnung der Kandidatenlisten lokaler politischer Parteien für Einwanderer durchgesetzt, und zwar häufig von oben, durch starke etablierte Eliten - und nicht in Reaktion auf eine breite Mobilisierung der Einwanderer selbst.

Hypothesenartig lassen sich drei Modelle der Inkorporation von Einwanderern in den politischen Mainstream skizzieren: Frankreich steht offenbar für ein Modell, in dem eine Inkorporation der Einwanderer typischerweise in Kooperation mit oder sogar entscheidend gestützt durch etablierte Kräfte erfolgt: So wurden erste Wahlerfolge vor allem aus dem Maghreb stammender Kandidat(inn)en in den späten 1980er Jahren durch die von der Sozialistischen Partei unterstützte Kampagne „France Plus“ gefördert: Individuen mit Migrationsgeschichte wurden als Parlamentskandidat(inn)en kooptiert, um neue Wählerpotenziale zu erschließen (vgl. bereits Geisser 1997). Ein zweites Modell 
des Aufstiegs von Einwanderern in Parlamente scheint eine Konstellation zu repräsentieren, in der eine gesellschaftliche Bewegung (und damit ein mobilisiertes Wählerpotenzial) existiert, die eine Vertretung der Minderheiten und ihrer Interessen einfordert und auf etablierte politische Kräfte trifft, die aufgrund existierender Normen des Antirassismus und der Akzeptanz ethnischer Pluralität hierfür offen sind. Drittens schließlich ist ein Modell denkbar, in dem Einwanderergruppen - begünstigt durch eine starke Siedlungskonzentration, ein Wahlkreissystem und eine starke Mobilisierung und Geschlossenheit der eigenen Gruppe - eine parlamentarische Repräsentanz aus eigener Kraft durchsetzen. ${ }^{8}$

Die Forschung zur Repräsentanz von Migrant(inn)en in Parlamenten und den Bedingungen parlamentarischer Karrieren in europäischen Staaten nimmt (sehr) langsam zu. ${ }^{9}$ Nachdem Entwicklungen in Großbritannien bereits seit etlichen Jahren wissenschaftlich untersucht werden (Electoral Commission 2002; Anwar 1994; Le Lohe 1998; Saggar 1998, 2000; Norris/Lovenduski 1994; Solomos/Back 1995), liegen jetzt auch über Belgien, vor allem Brüssel (Martiniello 1998a; Jacobs et al. 2006b), die Niederlande und auch Frankreich (Geisser 1997; Wihtol de Wenden 1988, 1999) Forschungsarbeiten vor. ${ }^{10}$ Gerade vergleichende Aussagen und Typisierungen aber sind aufgrund der schmalen empirischen Basis bislang nur in Form erster Hypothesen möglich (vgl. auch Bird 2004; Gowricharn 2001; zu ,muslimischen Eliten“ Klausen 2006).

8 Sicherlich gibt es daneben auch eine individuelle Karriere von Menschen mit Migrationshintergrund, die weder besonders von Migrant(inn)en oder ethnischen Minderheiten unterstützt noch als deren Repräsentanten kooptiert werden.

9 Eine erste Annäherung an Profile und Karrierebedingungen u.a. politisch ,aktiver“ Migrant(inn)en (,first-generation immigrants who devote substantial time and energy to civic activities“, Vogel 2008: 77) bieten die Ergebnisse eines europaweiten Projekts mit Studierenden (Vogel 2008).

10 Der wohl beste Überblick über die Repräsentation der sogenannten „ethnic minorities“ in unterschiedlichen Parlamenten existiert für Großbritannien, wo etwa die local government association entsprechende Statistiken führt. Dort wurden auch bereits in den 1970er Jahren - und seitdem wiederholt - Wahlstudien speziell zu den ethnischen Minderheiten zugeordneten Wähler(inn)en durchgeführt bzw. Beteiligung und Repräsentation z.B. in der British General Election Study standardmäßig berücksichtigt. In Frankreich haben Geisser und Oriol (2001) die Repräsentanz von Einwanderern unter Kandidaten und Gewählten der Kommunalwahlen von 2001 untersucht. In den Niederlanden wurde seit Einführung eines kommunalen Wahlrechts für Ausländer(innen) 1985 deren politische Partizipation vor allem auf lokaler Ebene in einigen Studien erforscht, etwa am Amsterdamer IMES (vgl. Studien von van Heelsum, Tillie u. a.). Für Deutschland hat da Fonseca im Rahmen eines Dissertationsprojekts eine Datenbank aller Kandidat(inn)en bei Bundestagswahlen seit 1990 erstellt. Die Auswertung läuft noch, vgl. erste Thesen in da Fonseca 2006. Andreas M. Wüst $(2002 ; 2006)$ hat mit 1999 und 2000/2001 erhobenen Daten das Wahlverhalten und politische Einstellungen eingebürgerter Deutscher untersucht. Ein Gesprächsband von Mely Kiyak (2007) vermittelt interessante Einblicke in die Karrieren und Überzeugungen von zehn türkeistämmigen Parlamentariern in Deutschland. Am Max-Planck-Institut zur Erforschung multiethnischer und multireligiöser Gesellschaften laufen Forschungsarbeiten zur Ausbildung politischer Eliten und zur kommunalen Ebene (vgl. Schönwälder 2008 und www.mmg.mpg.de). 


\section{Politischer Protest}

International gilt ein Schwerpunkt der wissenschaftlichen Aufmerksamkeit eindeutig traditionellen Formen des politischen Engagements, also vor allem der Beteiligung von Migrant(inn)en und ihren Nachkommen bzw. Angehörigen ethnischer Minderheiten an Wahlen sowie ihrer Repräsentanz in Parlamenten. Verschiedentlich wird allerdings zu Recht betont, dass sich politische Partizipation nicht auf Wahlen beschränkt und Migrant(inn)en zudem nicht erst als Eingebürgerte politisch handeln und in die politischen Prozesse im Einwanderungsland eingreifen. Über die Hervorhebung der begrenzten Rechte, die Staaten Migrant(inn)en einräumen, werde verschiedentlich übersehen, dass durchaus auch fremden Staatsangehörigen vielfältige Handlungsmöglichkeiten offenstehen, die diese auch nutzen (z.B. Bilodeau 2008; aber auch schon Kemp et al. 2000: 95). Tatsächlich gab es auch in der Bundesrepublik, wie ein Buch von Bojadžijev (2008) jetzt in Erinnerung ruft, bereits in den 1960er und 1970er Jahren etliche Streiks, Demonstrationen, Proteste von Migrant(inn)en (kritisch zur Annahme der politischen Passivität der Migrant(inn)en auch von Oswald et al. 2003; Martiniello 2005: 4-5, bereits 1981 Miller). Die Beispiele umfassen den Widerstand organisierter koreanischer Frauen gegen das Auslaufen ihrer Aufenthaltsgenehmigungen Ende der 1970er Jahre, Mieterproteste in Frankfurt, Komitees gegen ein für Ausländer(innen) gekürztes Kindergeld, wie es sie 1974/75 in 19 Städten gab, und zum Teil ausführlich geschilderte linke Mobilisierungsbemühungen etwa durch eine „Gruppe Arbeitersache“. Auswahlkriterium ist hier die „Autonomie“ von Aktionen und Organisierung, wobei dies als Distanz zu Gewerkschaften und politischen Parteien - nicht aber zu kleineren linken Gruppen definiert ist. Eine systematische Analyse etwa der typischen Anlässe und Verlaufsformen von Protesten, der Charakteristika der Akteure, von Bündnisstrukturen, auch der längerfristigen Wirkungen, steht weiter aus. ${ }^{11}$ Rucht und Heitmeyer (2008) bieten hierfür eine Unterscheidung von Mobilisierungspotenzialen (Konflikte und Probleme, die als bedeutsam empfunden werden) und Mobilisierungsfaktoren (Wahrnehmung von Problemen als gemeinsame, Mobilisierungsstrukturen, externe Anlässe) an. Auch ihre thesenartige Skizze illustriert die Defizite, die in der empirischen Erforschung der Mobilisierungen - und in der Konsequenz auch in der theoretischen Verallgemeinerung im spezifischen Feld der Mobilisierung von Migrant(inn)en - bestehen.

Einen Vorschlag zu einer systematischen Identifikation der Bedingungen politischer Mobilisierung macht - bezogen auf europäische Bewegungen für die Legalisierung von Migrant(inn)en ohne Aufenthaltsrechte - Barbara Laubenthal (2007; vgl. auch Schwenken 2006). ${ }^{12}$ Vor allem in Frankreich, aber auch 2001 in Spanien, bildeten sich solche Protestbewegungen, die durch z. T. spektakuläre Aktionsformen Zugang zur politischen

11 Bojadžijev geht es vor allem um die Bekräftigung einer Theorie des „relationalen Rassismus“, weniger eine systematische Erklärung von Verlauf und Formen politischen Protests. Zu den weiter bestehenden Desiderata der Forschung gehört auch eine Verbreiterung der empirischen Basis, wobei u.a. Informationen über Mobilisierungen aus zeitgenössischen Publikationen quellenkritisch überprüft werden sollten.

12 Schwenken zweifelt, ob generelle Aussagen über die Möglichkeit einer Mobilisierung solcher „schwachen Interessen“ möglich sind; eine allgemeine „Logik des kollektiven Handelns irregulärer MigrantInnen“" gebe es nicht (2006: 323 f.). 
Öffentlichkeit fanden. Eher kleine Gruppen der zugewanderten Bevölkerung agierten hier, unterstützt von etablierten zivilgesellschaftlichen Akteuren, in Frankreich auch von berühmten Individuen - und diese Unterstützung eines breiten Umfeldes identifiziert Laubenthal (2007: 110-111, 234) als eine Voraussetzung für die Entstehung und Wirkung einer Bewegung illegaler Migrant(inn)en. Eine zweite Bedingung sei es, dass die Anwesenheit der Migrant(inn)en - entgegen ihrem offiziellen Rechtsstatus - als legitim dargestellt werden könne, und zwar vor allem unter Hinweis auf historisch begründete Ansprüche an die ehemaligen Kolonialherren (z.B. den Tod des Vaters in der Armee Frankreichs, vgl. Guiraudon 2004: 66). Drittens spielten die Medien eine entscheidende Rolle in der Ausbreitung, für die Wirkung und für die Legitimierung solcher Bewegungen. Für die besondere Mobilisierungsfähigkeit und öffentliche Wirkung der französischen Bewegung war wohl darüber hinaus eine zeitspezifische Konstellation entscheidend, vor allem die gleichzeitige Hochzeit der Antirassismusbewegung und die Bereitschaft von Sozialisten und Kommunisten, migrationspolitische Reformforderungen zu unterstützen - erneut ein Hinweis auf die notwendige Berücksichtigung solcher konkreten Konstellationen in der Forschung.

\section{Transnationale Mobilisierungen?}

Während die Forschung zur Teilhabe von Einwanderern an einem nationalen politischen Prozess und dessen Institutionen sich gerade in Europa erst langsam entwickelt, ist in den letzten Jahren sehr lebhaft darüber diskutiert worden, ob und in welchem Maß die Entwicklung „transnationaler“ Strukturen und Loyalitäten diesen nationalstaatlichen Bezugsrahmen infrage stellt. In besonderem Maß scheinen Migrant(inn)en das Neue der Globalisierung, der Relativierung nationalstaatlicher Begrenzungen und der Entstehung neuer, grenzüberschreitender Räume zu repräsentieren. Nun ist es keineswegs neu, dass Auswanderer ein anhaltendes Interesse an den politischen Entwicklungen im Heimatland zeigen, dass sie auch z.B. durch die finanzielle Unterstützung bestimmter Bewegungen und Kampagnen versuchen, in diese zu intervenieren. Rucht/Heitmeyer (2008) greifen hierfür den Begriff der Diaspora-Politik auf (im Anschluss an Fijalkowski/Gillmeister 1997); andere Autoren sprechen von Herkunftsland-bezogener Mobilisierung oder „homeland politics“ (Vertovec 2009). Häufig signalisiert die heute zunehmend verbreitete Beschreibung bekannter Phänomene als ,transnational“ zunächst eine veränderte Bewertung grenzüberschreitender Beziehungen, indem die Bindung an das Herkunftsland nicht mehr als rückwärtsgewandt, als verzögerte Integration im Aufnahmeland interpretiert, sondern nunmehr als zeitgemäßer Ausdruck postnationaler Orientierungen begriffen wird. ${ }^{13}$ Heute gibt es aber auch tatsächlich neue Bedingungen und Formen einer Mobilisierung von Migrant(inn)en in Herkunfts- und Aufnahmeland. Durch verbesserte Kommunikations- und Interaktionsmöglichkeiten (Internet, Reisen, Geldtransfers) sowie erweiterte Rechte, vor allem durch die Ausbreitung mehrfacher Staatsangehörigkeiten, aber auch durch erweiterte Wahlrechte, wird nicht nur das anhaltende

13 Vgl. dagegen aber Koopmans et al., die einer Bewertung von heimatlandbezogenen Aktivitäten als integrationshemmend zuneigen (2005: 107-108, 142). 
Interesse an politischen Entwicklungen im Heimatland gefördert, sondern werden vor allem - so Vertovec (2009: 118) - Handlungsmöglichkeiten erweitert. Portes/Rumbaut (2006: 130-131) heben darüber hinaus die Rolle der Herkunftsländer hervor, deren Haltung zu den „Diasporas“ sich verändert habe, indem sie mehr Interesse an ihnen zeigten und verstärkt versuchten, auf sie Einfluss zu nehmen. Durch diese Faktoren, so die These, erhalten alte und zum Teil neue Formen der Interaktion eine größere Dauerhaftigkeit und Wirkung (vgl. auch Portes et al. 2008).

Ihren empirischen Bezugspunkt haben Thesen zur Entwicklung transnationaler Formen politischer Partizipation vor allem in Forschungen zu in den USA lebenden Migrant(inn)en. Hier hat die Einführung eines im Ausland auszuübenden Wahlrechts für nicht im Heimatland lebende Dominikaner (seit 1997) und Mexikaner (seit 2006) neue Bedingungen für eine Einflussnahme und Mobilisierung der Auswanderer im Heimatland geschaffen. In Mexiko sind in den vergangenen Jahren Politiker aufgetreten, die ihre Erfahrungen und Kontakte als in den USA lebende Migranten zum politischen Potenzial machten, indem sie etwa in Mexiko damit warben, Investitionen der Auswanderer im Heimatland stimulieren zu können und indem sie in den USA unter den Bürger(inne)n mexikanischer Herkunft eine eigene Wählerbasis mobilisierten (Smith/Bakker 2005; Hoffmann 2008). Ob zwischen den USA und einigen wichtigen Herkunftsländern der eingewanderten Bevölkerung besondere Beziehungen bestehen - etwa durch die geographische Nähe, sehr enge wirtschaftliche (und politische) Abhängigkeiten und die Größe des in den USA lebenden Bevölkerungsanteils - oder vergleichbare Entwicklungen auch z.B. zwischen Deutschland und der Türkei denkbar sind, wäre genauer zu erforschen. Østergaard-Nielsen (2003) konstatierte auch für die Türkei einen sich insbesondere seit den 1990er Jahren abzeichnenden Perspektivenwechsel hin zur Anerkennung der Permanenz der Auswanderung, verbunden mit verstärkten Versuchen, das Potenzial der Emigranten auch politisch zu nutzen. So wurde 1995 in der Verfassung vorgesehen, die (offenbar sehr bescheidene) Wahlbeteiligung im Ausland lebender türkischer Staatsangehöriger zu erleichtern. 2008 hat die türkische Regierung Schritte eingeleitet, um dies umzusetzen, indem eine Stimmabgabe im Ausland ermöglicht wird (vgl. Pressemeldungen, z.B. Tagesspiegel, 8. Jan. 2008). Der Auftritt des türkischen Regierungschefs Erdoğan in Köln im Februar 2008 vor in Deutschland lebenden Türk(inn)en dürfte hier seinen Hintergrund haben und vielleicht eine neue Präsenz türkischer Politik in Deutschland und auslandstürkischer Wählerpotenziale in der Türkei andeuten. $^{14}$

Aber es wäre falsch, nur auf die Türkei und die Türkeistämmigen zu blicken. In Kroatien galten 2007 die im Ausland lebenden kroatischen Staatsangehörigen als wahlentscheidender Faktor bei den Parlamentswahlen (vgl. z.B. www.wieninternational.at; 27.11.2007). Eine kroatische Exilpolitik gab es in der Bundesrepublik über Jahrzehnte,

14 Erdoğan äußerte dort u.a., die „türkische Gemeinschaft“" sollte in der Lage sein, „,in der deutschen politischen Landschaft einen Einfluss auszuüben“; sie sollte Bürgermeister stellen, „Vertreter und Gruppen" in den politischen Parteien haben, als Lobby für die türkischen Interessen tätig werden. Er verwendete dabei immer die ,wir“-Form. Andererseits versprach er, die Regierung der Türkei werde sich stärker mit den Interessen der im Ausland lebenden Türk(inn)en beschäftigen und sich für diese engagieren (vgl. die Übersetzung auf www.welt.de, 11. Februar 2008). 
vor allem bis in die sechziger Jahre (vgl. Clarkson 2008). Auch Italien gewährt seit 2001 seinen im Ausland lebenden Staatsangehörigen ein Wahlrecht im Ausland. In vier Wahlbezirken wählen diese aus ihrer Mitte, also aus dem Kreis der im Ausland lebenden Italiener(innen), sechs Senator(inn)en und zwölf Abgeordnete. Die Konjunktur des Transnationalismus in der Forschung hat die Aufmerksamkeit für solche zum Teil lange wenig beachteten Phänomene gestärkt. Systematischer untersucht werden sollte nun, unter welchen Bedingungen etwa ja keineswegs außergewöhnliche Wahlrechte im Ausland lebender Staatsangehöriger ${ }^{15}$ systematisch und auf neue Art durch als Gruppe mobilisierte Migrant(inn)en genutzt werden. Diskutiert werden sollte darüber hinaus, ob die heute vielfach sehr großzügige Verwendung des Ausdrucks „transnational“ als im Grunde synonym für „grenzüberschreitend“ hilfreich ist. Denkbar wäre es etwa, ihn zu reservieren für Phänomene, in denen der nationalstaatliche Rahmen überwunden oder relativiert wird (und nicht nur politisches Engagement in zwei Nationalstaaten stattfindet), indem sich ,transnationale“ soziale Felder und post-nationale Identitäten ausbilden. Portes et al. schlagen etwa vor, die transnationale Perspektive auszurichten auf eine anhaltende Interaktion von Herkunfts- und Aufnahmelandkontexten und die hieraus entstehenden komplexen, nationale Grenzen überwindenden sozialen Felder (Portes et al. 2008: 2; vgl. auch Faist/Özveren 2004).

Typisch scheint heute zu sein, dass „transnationale“ politische Aktivitäten doch insofern den nationalstaatlichen Kontext nicht überwinden, als sie Verknüpfungen zwischen dem aktuellen Lebensmittelpunkt und dem Herkunftsstaat repräsentieren. Ob auch in einem weiteren Sinne transnationale Organisations- und Bewegungsstrukturen entstehen könnten, wurde gelegentlich bezogen auf den Kontext der europäischen Integration oder auch eine Bewegung von Migrant(inn)en ohne Aufenthaltsstatus gefragt; die Befunde sind aber eher negativ. So hatten Versuche einer Zusammenführung von Migranten- und Antirassismusverbänden auf europäischer Ebene bislang nur äußerst begrenzten Erfolg (Schwenken 2006: 157-165). Guiraudon stellt zwar eine transnationale oder europäische Dimension z. B. der Illegalenbewegung fest, die sich in ,sporadic international political exchanges as well as instances of cross-border diffusion through press coverage“ äußere. Insgesamt aber blieben solche Bewegungen doch vor allem national fokussiert: „nation-based frames of reference and limited resources limit the extent of transnational activity that a grassroots movement can engage in“"(2004: 66-67). Auch Koopmans et al. (2005: 128) zeigen eine - zumindest soweit in der nationalen Presse berichtet - überragende Bedeutung des nationalen Rahmens als Bezugspunkt von migrations- und integrationspolitischen Forderungen der 1990er Jahre.

Der Umfang transnationalen (oder bi-nationalen) Engagements von Einwanderern wird nach anfänglich verbreiteter Euphorie in der Forschung heute vorsichtiger eingeschätzt. So kamen Guarnizo, Portes und Haller (2008: 2) für die USA zu dem Ergebnis, „that immigrants involved in transnational economic, political and socio-cultural activities represent a minority of their respective communities“. Selbst bei den Latinos bleibt ein transnationales Engagement die Ausnahme (DeSipio 2006). Gleichzeitig heben Portes et al. hervor, dass es sich beim ,immigrant transnationalism“ zwar überwiegend um ein

15 Ein 2007 erschienenes Handbuch (IDEA 2007) nennt 115 Staaten, in denen - in unterschiedlichem Maß und in unterschiedlich auszuübender Form - externe Wahlrechte bestehen. 
Phänomen der ersten Generation, nicht aber der Neuankömmlinge handle. Transnational politisch engagiert seien die stärker etablierten und daneben die besser gebildeten Einwanderer (Portes et al. 2008: 2, 6, 30). Darüber hinaus bestätigen Forschungen in den USA einen auch für Migrantenvereine in Deutschland bekannten Befund, wonach Interesse und Engagement in Heimatland- bzw. Einwanderungsland-bezogenen Kontexten zumeist keine Alternativen sind (Sauer/Halm 2004: 550; Salentin 2004). Zumindest für Latinos in den USA wurde gezeigt, dass Personen, die in Heimatland-bezogenen Organisationen aktiv sind, sich oft gleichzeitig auch in den USA engagieren, dass, wer an Wahlen im Herkunftsland teilnimmt, häufiger auch in den USA wählt (DeSipio 2006: 118).

\section{Bilanz}

Der politischen Inkorporation von Einwanderern, also deren Mitwirkung an den Entscheidungen über die Entwicklung der Gesellschaft, kommt eine zentrale Bedeutung im Rahmen eines auf gleichberechtigte Teilhabe zielenden Integrationsprozesses zu. Gerade in heterogenen Gesellschaften könnte eine Integration mittels Teilnahme an den politischen Kommunikations- und Entscheidungsprozessen immer wichtiger werden. Zu Verlauf und Mechanismen solcher Inkorporationsprozesse liegen in der internationalen Forschung einige Erkenntnisse vor. Es bestehen aber auch bedeutende Lücken, so etwa - wie in diesem Bericht hervorgehoben - im Hinblick auf die Analyse der Rolle politischer Institutionen, der Herausbildung von politischem Führungspersonal aus der eingewanderten Bevölkerung und der Mechanismen nicht-wahlpolitischer Mobilisierungen. Gleichzeitig zeichnen sich Entwicklungen ab, aus denen neue Fragen an die Forschung hervorgehen: Institutionen, die einmal wesentlich für eine politische Sozialisation von Einwanderern sorgten, erreichen diese vielleicht nicht mehr im bisherigen Umfang. Formen politischer Mobilisierung verändern sich, transnationale Bindungen und Aktionsformen könnten typische Inkorporationsprozesse von Einwanderern verändern. Für die breitere Entwicklung eines einschlägigen Forschungsfeldes auch in und über Deutschland gibt es also genug Stoff.

\section{Literatur}

Anwar, Muhammad. 1994. Race and Elections. Coventry: Centre for Research in Ethnic Relations.

Barreto, Matt A. und José A. Muñoz. 2003. Reexamining the „Politics of In-Between“: Political Participation Among Mexican Immigrants in the United States. Hispanic Journal of Behavioral Sciences 25: 427-447.

Bauböck, Rainer (Hrsg.). 2006. Migration and Citizenship. Legal Status, Rights and Political Participation. Amsterdam: Amsterdam University Press.

Berger, Maria, und Ruud Koopmans. 2004. Bürgerschaft, ethnische Netzwerke und die politische Integration von Türken in Amsterdam und Berlin. Forschungsjournal Neue Soziale Bewegungen 17: 70-79. 
Bilodeau, Antoine. 2008. Immigrants' Voice through Protest Politics in Canada and Australia: Assessing the Impact of Pre-Migration Political Repression. Journal of Ethnic and Migration Studies 34: 975-1002.

Bird, Karen. 2004. The Political Representation of Women and Ethnic Minorities in Established Democracies. A Framework for Comparative Research. AMID Working Paper, Alborg.

Bloemraad, Irene. 2006. Becoming a Citizen: Incorporating Immigrants and Refugees in the United States and Canada. Berkeley: University of California Press.

Bojadžijev, Manuela. 2008. Die windige Internationale: Rassismus und Kämpfe der Migration. Münster: Verlag Westfälisches Dampfboot.

Clarkson, Alexander. 2008. Home and Away: Immigration and Political Violence in the Federal Republic of Germany 1945 - 1990. Cold War History 8: 1-21.

De la Garza, Rodolfo O. 2004. Latino Politics. Annual Review of Political Science 7: 91-123.

DeSipio, Louis. 2001. Building America, One Person at a Time: Naturalization and the Political Behavior of the Naturalized in Contemporary American Politics. In: Gary Gerstle und John Mollenkopf (Hrsg.), E Pluribus Unum? Contemporary and Historical Perspectives on Immigrant Political Incorporation. New York: Russell Sage, 67-106.

DeSipio, Louis. 2006. Do Home-Country Political Ties Limit Latino Immigrant's Pursuit of U.S. Civic Engagement and Citizenship? In: Taeku Lee, S. Karthick Ramkrishnan und Ricardo Ramírez (Hrsg.), Transforming Politics, Transforming America. The Political and Civic Incorporation of Immigrants in the United States. Charlottesville/London: University of Virginia Press, 106-126.

Electoral Commission. 2002. Voter engagement among black and ethnic minority communities. Research report. London (www.electoralcommission.org.uk).

Faist, Thomas, und Eyüp Özveren (Hrsg.). 2004. Transnational social spaces: Agents, Networks, and Institutions. Aldershot: Ashgate.

Fennema, Meindert, und Jean Tillie. 2001. Civic community, political participation and political trust of ethnic groups. Connections 24: 26-41.

Fijalkowski, Jürgen, und Helmut Gillmeister. 1997. Ausländervereine - Ein Forschungsbericht. Berlin: Hitit.

Fonseca, Sara Claro da, 2006: Neue Bürger - neue Kandidaten? Die Parteien im Wettstreit um Migrantenstimmen,. WZB-Mitteilungen 114: 32-35.

Frey, William F. 2008. Race, Immigration and America's Changing Electorate. University of Michigan. Population Studies Center. Report 08-635. Ann Arbor.

Garbaye, Romain. 2005. Getting into Local Power: The Politics of Ethnic Minorities in British and French Cities. Oxford: Blackwell.

Geisser, Vincent 1997. Ethnicité républicaine: les élites d'origine maghrébine dans le système politique français. Paris: Presses de Sciences Po.

Geisser, Vincent, und Paul Oriol. 2001. Les Français „d'origine étrangère“ aux élections municipales de 2001: vers une normalisation de leur présence parmi les candidats et les élus? Migrations Société 13: 41-55.

Gowricharn, Ruben. 2001. Ethnic Minorities and Elite Formation. Journal of International Migration and Integration 2: 155-167.

Guarnizo, Luis Eduardo, Alejandro Portes und William Haller. 2003. Assimilation and Transnationalism: Determinants of Transnational Political Action among Contemporary Migrants. American Journal of Sociology 108: 1211-1248.

Guiraudon, Virginie. 2004. Ethnic Migrant Minorities and Transnational Claims-Making in Europe. Opportunities and Constraints. In: Flemming Christansen und Ulf Hedetoft (Hrsg.), The Politics of Multiple Belonging. Ethnicity and Nationalism in Europe and East Asia. Aldershot/Burlington: Ashgate, 61-75. 
Hoffmann, Bert. 2008. Exit and Voice in Transnational Times: Revisiting a Classic. Unveröffentlichtes Konferenzpapier für die Tagung „Migration(en) und Entwicklung(en). Transformation von Paradigmen, Organisationen und Geschlechterordnungen“, Bielefeld. 10.-11. Juli 2008.

IDEA. 2007. Voting from Abroad. The International IDEA Handbook. Stockholm: IDEA.

Jacobs, Dirk, Hassan Bousetta, Andrea Rea, Marco Martiniello und Marc Swyngedouw. 2006. Qui sont les candidats aux élections Bruxelloises? Le profil des candidats à l'élection au parlement de la région de Bruxelles-Capitale du 13 Juin 2004. Louvain-la-Neuve: Bruylant.

Jacobs, Dirk, Karen Phalet und Marc Swyngedouw. 2006. Political Participation and Associational Life of Turkish Residents in the Capital of Europe. Turkish Studies 7: 145-161.

Jacobs, Dirk, und Jean Tillie. 2008. Social Capital and Political Participation of Immigrants in Europe. Key developments in empirical studies. In: Dita Vogel (Hrsg.), Highly Active Immigrants. A resource for European civil societies. Frankfurt am Main: Peter Lang, 47-58.

Jones-Correa, Michael. 2007. Ethnic Politics. In: Mary C. Waters, Reed Ueda und Helen B. Marrow (Hrsg.), The New Americans. A guide to immigration since 1965. Cambridge, Mass.: Harvard University Press, 189-201.

Kemp, Adriana, Rebeca Raijman, Julia Resnik und Silvina Schammah Gesser. 2000. Contesting the limits of political participation: Latinos and black African migrant workers in Israel. Ethnic and Racial Studies 23: 94-119.

Kiyak, Mely. 2007. 10 für Deutschland. Gespräche mit türkeistämmigen Abgeordneten. Hamburg: edition Körber-Stiftung.

Klausen, Jytte. 2006. Europas muslimische Eliten. Frankfurt am Main/New York: Campus.

Koopmans, Ruud, Paul Statham, Marco Giugni und Florence Passy. 2005. Contested Citizenship. Immigration and Cultural Diversity in Europe. Minneapolis/London: University of Minnesota Press.

Kühne, Peter, Nihat Öztürk und Klaus-W. West (Hrsg.). 1994. Gewerkschaften und Einwanderung: Eine kritische Zwischenbilanz. Köln: Bund-Verlag.

Laubenthal, Barbara. 2007. Der Kampf um Legalisierung. Frankfurt am Main/New York: Campus.

Le Lohe, Michel J. 1998. Ethnic minority participation and representation in the British electoral System. In: Shamit Saggar (Hrsg.), Race and British Electoral Politics. London: UCL Press.

Martiniello, Marco. 1998a. Foreign Origin Politicians in Belgium. In: Marco Martiniello (Hrsg.), Multicultural Policies and the State. Utrecht: Ercomer, 109-124.

Martiniello, Marco. 1998b. Les immigrés et les minorités ethniques dans les institutions politiques: ethnicisation des systèmes politiques européens ou renforcement de la démocratie? Revue Européenne des Migrations Internationales 14: 9-17.

Martiniello, Marco. 2005. Political Participation, Mobilisation and Representation of Immigrants and their Offspring in Europe. Willy Brandt Series of Working Papers in International Migration and Ethnic Relations 1, Malmö.

Miller, Mark J. 1981. Foreign Workers in Western Europe: An Emerging Political Force. New York: Praeger.

Norris, Pippa, und Joni Lovenduski. 1995. Political Recruitment. Gender, Race and Class in the British Parliament. Cambridge: Cambridge University Press.

Østergaard-Nielsen, Eva. 2003. Turkey and the 'Euro Turks': Overseas Nationals as an Ambigious Asset. In: Eva Østergaard-Nielsen (Hrsg.), International Migration and Sending Countries. Perceptions, Policies and Transnational Relations. Basingstoke/New York: Palgrave Macmillan, 77-98.

Oswald, Anne von, Karen Schönwälder und Barbara Sonnenberger. 2003. Einwanderungsland Deutschland: a New Look at its Post-war History. In: Rainer Ohliger, Karen Schönwälder 
und Triadafilos Triadafilopoulos (Hrsg.), European Encounters. Migrants, Migration and European Societies since 1945. Aldershot: Ashgate, 19-37.

Öztürk, Nihat. 2002. Partizipation von Migranten und Einwanderern in Betrieben und den Gewerkschaften - Das Beispiel der Industriegewerkschaft Metall. In: Andreas Treichler (Hrsg.), Wohlfahrtsstaat, Einwanderung und ethnische Minderheiten. Wiesbaden: Westdeutscher Verlag, 295-309.

Penninx, Rinus, und Judith Roosblad (Hrsg.). 2000. Trade Unions, Immigration and Immigrants in Europe 1960-1993. New York/Oxford: Berghahn.

Portes, Alejandro, Cristina Escobar und Renelinde Arana. 2008. Bridging the Gap: Transnational and Ethnic Organizations in the political Incorporation of Immigrants in the United States. Ethnic and Racial Studies 31: 1056-1090.

Portes, Alejandro, und Rubén G. Rumbaut. 2006. Immigrant America: A Portrait. Berkeley: University of California Press.

Ramakrishnan, S. Karthick. 2005. Democracy in Immigrant America: Changing Demographics and Political Participation. Stanford: Stanford University Press.

Rucht, Dieter, und Wilhelm Heitmeyer. 2008. Mobilisierung von und für Migranten. In: Roland Roth und Dieter Rucht (Hrsg.), Die sozialen Bewegungen in Deutschland nach 1945. Ein Handbuch. Frankfurt am Main: Campus, 573-592.

Saggar, Shamit. 2000. Race and Representation. Manchester: Manchester University Press.

Saggar, Shamit (Hrsg.). 1998. Race and British Electoral Politics. London: UCL Press.

Salentin, Kurt. 2004. Ziehen sich Migranten in ,ethnische Kolonien' zurück? In: Klaus J. Bade, Michael Bommes und Rainer Münz (Hrsg.), Migrationsreport 2004: Fakten - Analysen Perspektiven. Frankfurt am Main/New York: Campus.

Sauer, Martina, und Dirk Halm. 2004. Das Zusammenleben von Deutschen und Türken - Entwicklung einer Parallelgesellschaft? WSI-Mitteilungen 57: 547 -554.

Schönwälder, Karen. 2008. Trendsetter Obama? The Ascent of Immigrant Voters and Politicians in European States. Working Paper. Göttingen: Max Planck Institute for the Study of Religious and Ethnic Diversity.

Schwenken, Helen. 2006: Rechtlos, aber nicht ohne Stimme. Politische Mobilisierungen und irreguläre Migration in die Europäische Union. Bielefeld: Transcript.

Skerry, Peter. 2005. Political institutions and minority mobility in the USA. In: Glenn C. Loury, Tariq Modood und Steven M. Teles (Hrsg.), Ethnicity, Social Mobility, and Public Policy. Comparing the USA and UK. Cambridge: Cambridge University Press, 475-497.

Smith, Michael Peter, und Matt Bakker. 2005. The transnational politics of the Tomato King: meaning and impact. Global Networks 5: 129-146.

Solomos, John, und Les Back. 1995. Race, Politics and Social Change. London/New York: Routledge.

Tillie, Jean. 2004. Social Capital of Organisations and their Members: Explaining the Political Integration of Immigrants in Amsterdam. Journal of Ethnic and Migration Studies 30: 529541.

Vertovec, Steven. 2009. Transnationalism. London: Routledge.

Vogel, Dita (Hrsg.). 2008. Highly Active Immigrants. A resource for European civil societies. Frankfurt am Main: Peter Lang.

Waldrauch, Harald, und Karin Sohler. 2004. Migrantenorganisationen in der Großstadt: Entstehung, Strukturen und Aktivitäten am Beispiel Wien. Frankfurt am Main: Campus.

Werz, Michael. 2008. Braun, Schwarz, Weiß. Die Amerikaner wählen und entdecken einen neuen Kontinent. Kommune 25: 25-27.

Wihtol de Wenden, Catherine. 1988. Les immigrés et la politique. Cent-cinquante ans d'évolution. Paris: Presses de la FNSP.

Wihtol de Wenden, Catherine. 1999. Post-1945 Migration to France and Modes of Socio-Political Mobilisation. IMIS-Beiträge 13: 43-73. 
Wong, Janelle S., Pei-te Lien und M. Margaret Conway. 2008. Activity amid Diversity: Asian American Political Participation. In: Jane Junn und Kerry L. Haynie (Hrsg.), New Race Politics in America. Understanding Minority and Immigrant Politics. Cambridge: Cambridge University Press, 70-94.

Wüst, Andreas M. 2002. Wie wählen Neubürger? Politische Einstellungen und Wahlverhalten eingebürgerter Personen in Deutschland. Opladen: VS Verlag für Sozialwissenschaften.

Wüst, Andreas M. 2006. Wahlverhalten und politische Repräsentation von Migranten. Der Bürger im Staat 56: 228-234. 